\title{
ELEMENTS OF FINITE ORDER IN STONE-ČECH COMPACTIFICATIONS
}

\author{
by JOHN BAKER, NEIL HINDMAN* and JOHN PYM
}

(Received 14th November 1990)

\begin{abstract}
Let $S$ be a free semigroup (on any set of generators). When $S$ is given the discrete topology, its Stone-Cech compactification has a natural semigroup structure. We give two results about elements $p$ of finite order in $\beta S$. The first is that any continuous homomorphism of $\beta S$ into any compact group must send $p$ to the identity. The second shows that natural extensions, to elements of finite order, of relationships between idempotents and sequences with distinct finite sums, do not hold.
\end{abstract}

1980 Mathematics subject classification (1985 Revision): 22A15

\section{Introduction}

One of the unsolved problems about the semigroup $\beta \mathbb{N}$ is whether it contains an element of finite order, that is to say a $p$ such that $p+p+\cdots+p=p$ where there cannot be fewer than three terms on the left-hand side. Here we present two main results about elements of finite order which the sceptical reader may interpret as evidence that they do not exist. The first is easy to state: any continuous homomorphism from $\beta \mathbb{N}$ to a compact topological group must send each element of finite order to the zero of the group. The second concerns the relationship between elements of finite order and finite sums of terms in sequences. The parallel with the theory of idempotents necessarily fails-see Theorem 3.2. In order to make the discussion of finite sums more natural, we broaden our context from $\beta \mathbb{N}$ to compactifications of general free semigroups.

We introduce our notation and some basic known results. The operation in all semigroups, whether commutative or not, will be denoted by + and we shall use additive terminology in all respects save one: an idempotant $p$ satisfies $p+p=p$. A sum $p+p+\cdots+p$, with $k$ terms, is denoted by $k p$. To say $p$ has order $k$ is to say $(k+1) p=p$ and $r p \neq p$ if $r \leqq k$; it is easy to see that this implies that $k p$ is idempotent and $\{p, 2 p, \ldots, k p\}$ is a group.

If $S$ is a discrete semigroup than the Stone-Cech compactification $\beta S$ (which we regard as containing $S$ ) has a unique semigroup operation + with the properties (i) $x \rightarrow x+y$ is continuous on $\beta S$ for each $y \in \beta S$ and (ii) $y \rightarrow s+y$ is continuous on $\beta S$ for each $s \in S$. Since $S$ is dense in $\beta S$ the sum $x+y$ of $x, y \in \beta S$ is determined by $S$ and the following consequence of (i) and (ii): (iii) if $\left(s_{\alpha}\right),\left(t_{\beta}\right)$ are nets in $S$ with $s_{\alpha} \rightarrow x, t_{\beta} \rightarrow y$ in $\beta S$

*This author gratefully acknowledges support received from the National Science Foundation via grant DMS-8901058 and DMS-9025025. 
then $x+y=\lim _{\alpha} \lim _{\beta}\left(s_{\alpha}+t_{\beta}\right)$ (and the order of the limits here is crucial). From (iii) it is easy to deduce (iv) if $f: S \rightarrow T$ is a homomorphism of discrete semigroups then the unique continuous extension $f^{\beta}: \beta S \rightarrow \beta T$ is a homomorphism, and (v) if $g: S \rightarrow K$ is a homomorphism into a compact separately continuous semigroup $K$ than $g^{\beta}: \beta S \rightarrow K$ is a homomorphism.

We shall need two facts about a compact jointly continuous semigroup $K$. The first is immediate from joint continuity: (vi) if $x_{\alpha} \rightarrow x$ in $K$ then $k x_{\alpha} \rightarrow k x$ for any positive integer $k$. The second is well known in compact semigroup theory. (vii) If $K$ is a group and $S$ is a commutative subsemigroup, then $\mathrm{cl}_{K} S$ is a group. (Proof. The $\operatorname{closure} \mathrm{cl}_{K} S$ is a commutative semigroup since $S$ is, and it is compact, therefore its minimal ideal is a group $G$. The idempotent in $G$ can only be the zero of $K$, and hence (as $G$ is an ideal) $G=\mathrm{cl}_{K} S$.)

(Standard results about compact semigroups in general and $\beta S-$ or, more particularly, $\beta \mathbb{N}-$ can be found in many places, e.g. $[1,4,6,11]$.)

\section{Images of elements of finite order}

Let $X$ be a set. Let $F(X)$ be the free semigroup on the set $X$ of generators with the discrete topology. In this section we fix $k \in \mathbb{N}$ and fix $p \in \beta F(X)$ with the property that $k p$ is idempotent (so $p$ could be of order $k$ ).

Lemma 2.1. Let $K$ be a compact commutative jointly continuous semigroup. Let $f: F(X) \rightarrow K$ be a homomorphism and suppose that every element of $f(X)$ is divisible by $k$ (that is, for each $x \in X$ the equation $k y=f(x)$ has at least one solution $y$ in $K$ ). Then $f^{\beta}(p)$ is idempotent.

Proof. For each $x \in X$ pick $\bar{x} \in K$ with $k \bar{x}=f(x)$. Define $g: F(X) \rightarrow K$ to be the unique homomorphism satisfying $g(x)=\bar{x}$ for $x \in X$. For any $x_{1}, \ldots, x_{r} \in X$ we then have (using the commutativity of $K$ for the second equality)

$$
\begin{aligned}
k g\left(x_{1}+\cdots+x_{r}\right) & =k\left(g\left(x_{1}\right)+\cdots+g\left(x_{r}\right)\right)=k g\left(x_{1}\right)+\cdots+k g\left(x_{r}\right) \\
& =f\left(x_{1}\right)+\cdots+f\left(x_{r}\right)=f\left(x_{1}+\cdots+x_{r}\right),
\end{aligned}
$$

that is, $k g(x)=f(x)$ for $x \in F(X)$. Taking limits using $1\left(\right.$ vi) gives $k g^{\beta}(x)=f^{\beta}(x)$ for $x \in \beta F(X)$. By $1(\mathrm{v}), g^{\beta}$ is a homomorphism, so $k g^{\beta}(x)=g^{\beta}(k x)$ for $x \in \beta F(X)$. Since $k p$ is idempotent, so is $g^{\beta}(k p)=f^{\beta}(p)$.

Theorem 2.2. Let $G$ be a compact commutative topological group and let $f: F(X) \rightarrow G$ be a homomorphism. Then $f^{\beta}(p)=0$, the zero of $G$.

Proof. If $G=T$, the circle group, Lemma 2.1 gives the result immediately. Otherwise, by the Pontjagin Duality Theorem [3, Theorem 24.8], $G$ is embeddable in some product 
$T^{I}$ of circle groups. Following $f$ by the projection into the $i$ th component of this product shows that the $i$ th coordinate of $f^{\beta}(p)$ is 0 . So $f^{\beta}(p)$ is 0 .

Corollary 2.3. If $G$ is any compact topological group and $f: \mathbb{N} \rightarrow G$ is a homomorphism then $f^{\beta}(p)=0$ if $p$ has order $k$ in $\beta \mathbb{N}$.

Proof. Since $f(\mathbb{N})$ is a commutative subsemigroup of $G$, i(vii) shows that $\operatorname{cl}_{G} f(\mathbb{N})=$ $f^{\beta}(\beta \mathbb{N})$ is a commutative group. Theorem 2.2 applies to $f: \mathbb{N} \rightarrow f^{\beta}(\beta \mathbb{N})$ since $\mathbb{N}$ is the free semigroup on one generator.

Corollary 2.4. For $w \in F(X)$ denote by $l(w)$ the length of the word $w$. Let $m \in \mathbb{N}$ be given. Let $\left(w_{\alpha}\right)$ in $F(X)$ satisfy $w_{\alpha} \rightarrow p$. Then $l\left(w_{\alpha}\right)$ is eventually a multiple of $m$.

Proof. Write $l_{m}(w)=l(w)(\bmod m)$, so that $l_{m}: F(X) \rightarrow \mathbb{Z}_{m}=\mathbb{Z} / m \mathbb{Z}$. Since $l$ is a homomorphism into $\mathbb{N}, l_{m}$ is also a homomorphism. Theorem 2.2 tells us that $l_{m}^{\beta}(p)=0$. Since $l_{m}\left(w_{\alpha}\right) \rightarrow l_{m}^{\beta}(p)=0$ and $\mathbb{Z}_{m}$ is discrete, eventually $l_{m}\left(w_{\alpha}\right)=0$, as required.

\section{Finite sums}

If $\left\langle x_{n}\right\rangle_{n=1}^{\infty}$ is a sequence in a semigroup $S$ we write $F S\left\langle x_{n}\right\rangle_{n=1}^{\infty}$ (or simply $F S\left\langle x_{n}\right\rangle$ ) for $\left\{x_{n_{1}}+\cdots+x_{n_{r}}: r \in \mathbb{N}, n_{1}<n_{2}<\cdots<n_{r}\right\}$, the set of finite sums from $\left\langle x_{n}\right\rangle$. We say $\left\langle x_{n}\right\rangle$ has distinct finite sums if $x_{m_{1}}+\cdots+x_{m_{r}}=x_{n_{1}}+\cdots+x_{n_{s}}$, with the suffices on each side increasing, implies $r=s$ and $m_{1}=n_{1}, \ldots, m_{r}=n_{r}$.

Under certain circumstances, it is easy to find sequences with distinct finite sums. For example, if $S$ is cancellative every sequence $\left\langle x_{n}\right\rangle$ of distinct elements has a subsequence with distinct finite sums. (Proof. For any $y, x$ in a cancellative $S$, the set $-y+x=$ $\{z: x=y+z\}$ contains either no elements or just one. Given $N \in \mathbb{N}$, the sets

$$
\Sigma_{N}=\left\{x_{n_{1}}+\cdots+x_{n_{r}}: n_{1}<\cdots<n_{r} \leqq N\right\}, \quad \Delta_{N}=\cup\left\{-y+x: y, x \in \Sigma_{N}\right\}
$$

are finite. We can therefore find $x_{m} \in\left\langle x_{n}\right\rangle \backslash\left(\Sigma_{N} \cup \Delta_{N}\right)$ with $m>N$. Then for any $x, y \in \Sigma_{N}$ both $x \neq x_{m}$ and $x \neq y+x_{m}$, that is, no sum involving $x_{m}$ and $x_{n}$ 's with $n \leqq N$ can be equal to a sum in $\Sigma_{N}$. It is now easy to see how to produce inductively a subsequence $\left\langle x_{n_{r}}\right\rangle$ with distinct finite sums, starting with $x_{n_{1}}=x_{1}$.)

When $X=\left\{x_{1}, x_{2}, \ldots\right\}, F S\left\langle x_{n}\right\rangle$ in $F(X)$ is called an abstract distinct finite sum system (or ADFSS); as $F(X)$ is free on $X$, these finite sums are obviously distinct. When $S$ is a semigroup, a map $f: X \rightarrow S$ extends in a unique way to a homomorphism, which we again denote by $f$, of $F(X)$ into $S$, and obviously $F S\left\langle x_{n}\right\rangle$ maps onto $F S\left\langle f\left(x_{n}\right)\right\rangle$. The sequence $\left\langle f\left(x_{n}\right)\right\rangle$ has distinct finite sums if and only if $f$ is injective on $F S\left\langle x_{n}\right\rangle$. One point of considering ADFSS's (which were called non-commutative oids in [9]) is that in many situations $f$ is injective on $F S\left\langle x_{n}\right\rangle$ but not on $F(X)$ (see the examples in [10]). In such cases concepts applicable to free semigroups can sometimes be used in the ADFSS. For example length, $l$, makes sense for elements of $F S\left\langle f\left(x_{n}\right)\right\rangle$ if $f$ is injective on $F S\left\langle x_{n}\right\rangle$. 
When $f: F(X) \rightarrow S$ is a homomorphism and $F(X)$ and $S$ have their discrete topologies, then by 1 (iv) $f^{\beta}$ is a homomorphism from $\beta F(X)$ to $\beta S$. If in addition the restriction of $f$ to $F S\left\langle x_{n}\right\rangle$ is injective, then $f^{\beta}$ is bijective from $\operatorname{cl}_{\beta F(X)} F S\left\langle x_{n}\right\rangle$ into $\operatorname{cl}_{\beta S} F S\left\langle f\left(x_{n}\right)\right\rangle$, and therefore $f^{\beta}$ is an isomorphism of whatever algebraic structure $\beta F(X)$ induces on $\mathrm{cl}_{\beta F(X)} F S\left\langle x_{n}\right\rangle$. Now in fact there is a considerable amount. Write $H=\bigcap_{r} \mathrm{cl}_{\beta F(X)}$ $F S\left\langle x_{n}\right\rangle_{n=r}^{\infty}$. If $u \in F S\left\langle x_{n}\right\rangle_{n=r}^{\infty}$ then so is $u+w$ whenever $w \in F S\left\langle x_{n}\right\rangle_{n=s}^{\infty}$ and $s$ is greater than the largest suffix occurring in the sum for $u$. Using 1(ii) we conclude that $u+H \subseteq \mathrm{cl}_{\beta F(X)} F S\left\langle x_{n}\right\rangle_{n=r}^{\infty}$. Taking limits again using 1(i) gives $H+H \subseteq H$, that is, $H$ is a subsemigroup of $\beta F(X)$. Thus $f^{\beta}$ is an isomorphism of $H$ onto a subsemigroup of $\beta S$.

Proposition 3.1. Let $S$ be a semigroup, and let $\left\langle y_{n}\right\rangle$ be a sequence in $S$ with distinct finite sums. Define a homomorphism $f: F(X) \rightarrow S$ by writing $f\left(x_{n}\right)=y_{n}$ for all $n$. If $p \in f^{\beta}(\mathrm{H})$ is of finite order, $\left(w_{\alpha}\right)$ is a net in $F S\left\langle y_{n}\right\rangle$ and $w_{\alpha} \rightarrow p$, then for any given positive integer $m$ the length $l\left(w_{\alpha}\right)$ is eventually a multiple of $m$.

Proof. This is immediate from Corollary 2.4 and the above remarks.

A long time ago some basic relationships between idempotents and finite sums were established. To describe them, let $S$ be a discrete semigroup. An element $p \in \beta S$ will be considered to be an ultrafilter on $S$, so that $A \in p$ implies that $\mathrm{cl}_{\beta S} A$ is a neighbourhood of $p$ in $\beta S$ and, on the other hand, if $V$ is a neighbourhood of $p$ in $\beta S$ then $V \cap S \in p$. Then

(1) if $p$ is idempotent and $A \in p$ there is $\left\langle x_{n}\right\rangle$ with $F S\left\langle x_{n}\right\rangle \subseteq A$, (see[7]) and

(2) for every sequence $\left\langle x_{n}\right\rangle, \bigcap_{m} \operatorname{cl}_{\beta S} F S\left\langle x_{n}\right\rangle_{n=m}^{\infty}$ contains idempotents (and so if $p$ is such an idempotent $F S\left\langle x_{n}\right\rangle \in p$ ), see [5].

Recently a new link between (1) and (2) was found.

(3) It is consistent with ZFC that there are idempotents $p$ such that for each $A \in p$ it is possible to find $\left\langle x_{n}\right\rangle$ satisfying (1) and also $p \in \mathrm{cl}_{\beta S} F S\left\langle x_{n}\right\rangle$.

(3) has been proved using Martin's Axiom [7]. It has also been shown $[2,8]$ that (3) is independent of ZFC.

We might expect there to be analogues of these results for elements of order $k$. Write $F S_{i(\bmod k)}\left\langle x_{n}\right\rangle$ for the subset of $F S\left\langle x_{n}\right\rangle$ consisting of sums of the form $x_{n_{1}}+\cdots+x_{n_{r}}$ with $r \equiv i(\bmod k)$. Then these two conjectures seem natural.

$\left(1_{k}\right)$ If $p \in \beta S$ has order $k$ and $A_{i} \in i p$ for $1 \leqq i \leqq k$, there is a sequence $\left\langle x_{n}\right\rangle$ with $F S_{i(\bmod k)}\left\langle x_{n}\right\rangle \subseteq A_{i}$ for each $i$.

$\left(2_{k}\right)$ For every sequence $\left\langle x_{n}\right\rangle, \bigcap_{m} \operatorname{cl}_{\beta S} F S_{1(\bmod k)}\left\langle x_{n}\right\rangle_{n=m}^{\infty}$ contains elements of order $k$.

More generally, we might expect $\bigcap_{m} \mathrm{cl}_{\beta S} F S_{i(\bmod k)}\left\langle x_{n}\right\rangle_{n=m}^{\infty}$ to contain an element whose order is the order of $i$ in the group of integers $\bmod k$. In fact, we have the following result. 
Theorem 3.2. $\left(1_{k}\right)$ is true but $\left(2_{k}\right)$ is not. Indeed, for any sequence $\left\langle x_{n}\right\rangle$ with distinct finite sums, $\bigcap_{m} \operatorname{cl}_{\beta S} F S_{i(\bmod k)}\left\langle x_{n}\right\rangle_{n=m}^{\infty}$ contains no element of finite order if $i \neq 0(\bmod k)$.

Proof. As we wish to end on a positive note, we first consider $\left(2_{k}\right)$. Let $\left\langle x_{n}\right\rangle$ have distinct finite sums. Let $w_{\alpha} \in F S_{i(\bmod k)}\left\langle x_{n}\right\rangle, w_{\alpha} \rightarrow p \in \bigcap_{r} \operatorname{cl}_{\beta S} F S_{i(\bmod k)}\left\langle x_{n}\right\rangle_{n=r}^{\infty}$, where $p$ has finite order. Then Proposition 3.1 tells us, in particular, that $l\left(w_{a}\right)$ is eventually a multiple of $k$, but in fact $l\left(w_{\alpha}\right)=i(\bmod k)$ for all $\alpha$.

We establish $\left(1_{k}\right)$ by the following inductive construction. We claim that we can define decreasing sequences $\left\langle V_{n}(i)\right\rangle_{n=0}^{\infty}$ of neighbourhoods of $i p$, for $1 \leqq i \leqq k$, and a sequence $\left\langle x_{n}\right\rangle_{n=1}^{\infty}$ of elements of $S$ with the following properties:

$$
\begin{aligned}
V_{n}(i) \subseteq \mathrm{cl}_{\beta S} A_{i}, & x_{n} \in V_{n}(1), \\
x_{n}+i p \in V_{n-1}(i+1), \quad \text { and } & x_{m}+V_{n}(1) \subseteq V_{m-1}(i+1),
\end{aligned}
$$

for all relevant values of $i, m, n$ with $m \leqq n$. To see this, first choose $V_{0}(i)=\mathrm{cl}_{\beta S} A_{i}$ for each $i$. Next, choose $x_{1} \in S \cap V_{0}(1)$ satisfying the conditions that $x_{1}+i p \in V_{0}(i+1)$ for $1 \leqq i \leqq k$, where addition in $\{1,2, \ldots, k\}$ is taken as addition modulo $k$. Now suppose that we have chosen $V_{n}(i)$ and $x_{n+1}$ with the required properties, for $0 \leqq n \leqq r$ and $1 \leqq i \leqq k$. Since, for every $n=1,2, \ldots, r+1$ and $1 \leqq i \leqq k, x_{n}+i p \in V_{n-1}(i+1)$, it is possible, for each $i$, to choose a neighbourhood $V_{r+1}(i)$ of ip satisfying $V_{r+1}(i) \subseteq V_{r}(i)$, and $x_{n}+V_{r+1}(i) \subseteq$ $V_{n-1}(i+1)$ for all $n=1,2, \ldots, r+1$. Having done this, we then choose $x_{r+2} \in S \cap V_{r+1}(1)$ satisfying $x_{r+2}+i p \in V_{r+1}(i+1)$ for $1 \leqq i \leqq k$.

Having constructed these sequences, observe that if $n_{1}<n_{2}<\cdots<n_{r}$ then $x_{n_{1}}+x_{n_{2}}+$ $\cdots+x_{n_{r}} \in V_{n_{r}} \in V_{n_{1}-1}(r(\bmod k))$. From this it is clear that $\left\langle x_{n}\right\rangle$ has the required properties.

\section{Acknowledgement}

The authors would like to thank the referee for making improvements to the statement and proof of Theorem 3.2.

\section{REFERENCES}

1. J. F. Berglund, H. D. Junghenn and P. Milnes, Analysis on Semigroups (Wiley, New York, 1989).

2. A. Blass and N. Hindman, On strongly summable ultrafilters and union ultrafilters, Trans. Amer. Math. Soc. 304 (1987), 83-99.

3. E. Hewitt and K. A. Ross, Abstract Harmonic Analysis I (Springer, Berlin, 1979).

4. N. Hindman, Ultrafilters and combinatorial number theory (Lecture Notes in Mathematics 851, 1979), 119-184.

5. N. Hindman, The ideal structure of the space of $\kappa$-uniform ultrafilters on a discrete semigroup, Rocky Mountain J. Math. 16 (1986), 685-701. 
6. N. Hindman, Ultrafilters and Ramsey theory, -an update (Lecture Notes in Mathematics 1401, 1989), 97-118.

7. N. Hindman, Summable ultrafilters and finite sums, Contemp. Math. 65 (1987), 263-274.

8. P. Matet, Some filters of partitions, J. Symbolic Logic 53 (1988), 540-553.

9. T. Papazyan, Oids, finite sums and the structure of the Stone-Cech compactification of a discrete semigroup, Semigroup Forum, to appear.

10. J. S. Pym, Semigroup structure in Stone-Cech compactifications, J. London Math. Soc. 36 (1987), 421-428.

11. J. S. Pүм, Compact semigroups with one-sided continuity, in The Analytical and Topological Theory of Semigroups (ed. K. H. Hofmann et al., de Gruyter, Berlin, 1990), 197-217.

John Baker and John Pym

Department of Pure Mathematics

UNIVERSITY OF ShefFIELd

SHEFFIELD S3 7RH

ENGLAND
Neil Hindman

Department of Mathematics

Howard UNIVERSITY

WASHINGTON DC 20059

USA 